\title{
Horta comunitária como atividade lúdica para idosos institucionalizados
}

\section{Community garden as a play activity for institutionalized elderly persons}

\author{
Francisco Glauber Peixoto Ferreira' $\bullet$ Cácia Aline Costa Santos ${ }^{2} \bullet$ Carolina Maria de Lima Carvalho $^{3}$ \\ Vanessa Emille Carvalho de Souza Freire ${ }^{4}$
}

\begin{abstract}
RESUMO
Objetivo: relatar a experiência da implementação de uma horta comunitária, em uma Instituição de Longa Permanência para Idosos na capital cearense. Método: trata-se de relato de experiência sobre a implementação de uma horta comunitária em uma Instituição de Longa Permanência para Idosos, localizada na cidade de Fortaleza, Ceará, Brasil, realizada em 2018. A atividade foi desenvolvida por meio de integração interdisciplinar entre os Cursos de Graduação em Enfermagem e Agronomia de uma universidade pública. Resultados: a experiência vivenciada permitiu conhecer a personalidade dos moradores da instituição, histórias e aspirações destes, ao qual se pode implementar um ambiente lúdico e ao mesmo tempo produtivo para o estilo de vida desse idosos. Conclusão: a implementação da horta comunitária na instituição contribuiu de forma significativa para o bem-estar dos idosos, rompeu a monotonia e o sentimento de abandono vivenciados no dia a dia dos idosos institucionalizados e viabilizou o aproveitamento de um espaço inutilizado.

Palavras Chave: Instituição de Longa Permanência para Idosos; Jardinagem; Atenção à saúde do idoso.
\end{abstract}

\begin{abstract}
Objective: to report the experience of implementing a community garden in a Long Term Care Institution for the Elderly in the capital of Ceará. Method: this is an experience report on the implementation of a community garden in a Long Term Care Institution for the Elderly, located in the city of Fortaleza, Ceará, Brazil, held in 2018. The activity was developed through interdisciplinary integration between the Undergraduate Nursing and Agronomy courses at a public university. Results: the lived experience allowed to know the personality of the institution's residents, their histories and aspirations, to which a playful and at the same time productive environment can be implemented for the lifestyle of these elderly people. Conclusion: the implementation of the community garden in the institution contributed significantly to the well-being of the elderly, broke the monotony and the feeling of abandonment experienced in the daily lives of institutionalized elderly people and made it possible to take advantage of an unused space.
\end{abstract}

Keyword: Institution of Long Stay for the Elderly; Gardening; Health care for the elderly.

NOTA

I Enfermeiro, Mestrando pelo Programa de Pós-Graduação de Mestrado Acadêmico em Sociobiodiversidade e Tecnologia Sustentável pela Universidade da Integração Internacional da Lusofonia Afro-Brasileira - MASTS/UNILAB, CE. Contribuição: coleta de dados, redação do estudo, construção das discussão e resultados, revisão e aprovação final da versão publicada. Email: fgpf.glauber@hotmail.com https://orcid.org/0000-0002-3980-7253 http://lattes.cnpq.br/6898728780485440

2 Enfermeira pela Universidade da Integração Internacional da Lusofonia Afro-Brasileira - UNILAB, CE.Assistencialista da Estratégia Saúde da Família - ESF no Estado do Ceará. Contribuição: coleta de dados, redação do estudo, construção das discussão e resultados. Email: caciaaline@gmail.com https://orcid.org/0000-00022949-9234 http://lattes.cnpq.br/96329/5952040768

3 Enfermeira, Doutora e Mestre em Enfermagem pela Universidade Federal do Ceará -UFC, CE. Professora adjunta do curso de Enfermagem e da Especialização em Saúde da Família pela Universidade da Integração Internacional da Lusofonia Afro-Brasileira UNILAB. Possui experiência na área de Enfermagem, com ênfase em Oncologia Câncer de Mama. Contribuição: orientação, estruturação do estudo, construção das discussão e resultados, revisão e aprovação final da versão publicada. Email: carolinacarvalho@unilab.edu.br https://orcid.org/0000-0002-5 I73-5360 http://lattes.cnpq.br/2557330933945 I 07

4 Enfermeira, PHD em Enfermagem pela University of Illinois at Chicago, Estados Unidos, EUA. Doutora e Mestre em Enfermagem Pela Universidade Federal do Ceará - UFC, CE. Possui área de expertise na temática de acurácia diagnóstica, validação de diagnósticos de enfermagem, doenças cardiovasculares, doenças respiratórias, classificações de enfermagem, usabilidade e informática aplicada à enfermagem. Contribuição: orientação, estruturação do estudo, construção das discussão e resultados, tradução, revisão e aprovação final da versão publicada. Email: vanessaemille@gmail.com https://orcid.org/0000-0003-357/-0267 http:// lattes.cnpq.br/501964050759280। 


\section{INTRODUÇÃO}

As Instituições de Longa Permanência para Idosos (ILPI) se caracterizam como entidades de acolhimento coletivo e individual, com caráter domiciliar, que oferecem determinadas vertentes de cuidados, sejam de direitos sociais ou relacionados à saúde e ao bem-estar. Em contrapartida, esses ambientes funcionam como miniuniversos, uma vez que se criam relações interpessoais entre os residentes e a equipe atuante ${ }^{(1)}$.

Ao longo da história, esses espaços estavam ligados à natureza religiosa ou às ações de caridades, o que despertava, na visão social, paradigma de pobreza, abandono e solidão(2). Contudo, as ILPI ganharam novo formato assistencial no decorrer da história, em virtude de melhorias estruturais e organizacionais ${ }^{(3)}$. Também, observa-se não padronização quanto ao funcionamento dessas instituições, porém a legislação vigente preconiza que as instalações sejam adequadas e seguras para acomodar os idosos ${ }^{(4)}$.

Ainda nesse contexto, os espaços das ILPI no Brasil, em maioria, são constituídos por dormitórios, copa, direção e ambiente social, este último como alternativa única de atividades de lazer, no intuito de manter a interação entre os moradores institucionais. No entanto, há alternativas quanto à dinamização nesses espaços que, por meio de planejamento adequado, corrobora melhor qualidade de vida e recreação. A exemplo, citam-se os Espaços Verdes Urbanos (EVU), com justificativa de criar instalações de infraestrutura, de modo a inserir o idoso na própria manutenção da área e estética visual(5).

A relação com o meio é algo íntimo do ser humano, nesta questão, o conforto remete à ligação física e sentimental com o lugar que habita. Para tanto, por mais que não seja o recinto específico do ambiente familiar, a pessoa idosa começa a construir novas concepções, conceitos e história com o novo tipo de moradia. Ademais, a institucionalização significa relação de dependência, na medida em que o elo de confiança será estabelecido com base nas necessidades, nas limitações físicas e no manejo por profissionais denominados de cuidadores ${ }^{(6)}$.

Quanto à vivência, os idosos residentes possuem inter-relação com o ambiente e os sujeitos inseridos, o que gera um dia a dia compartilhado no âmbito social e comunitário, contudo, nota-se desfalque no tocante às atividades de caráter lúdico, de maneira que a rotina é composta de cuidados prestados por profissionais e diálogo entre os demais membros institucionalizados. Nesse contexto, cabe mencionar o surgimento das psicopatologias, como é o caso da depressão, que possui fatores multivariados e atinge, principalmente, idosos do sexo feminino, acompanhado de comorbidades ${ }^{(7)}$.

Sob tais constatações, as terapias ocupacionais funcionam como método paliativo no tratamento de pato- logias mentais, como depressão e ansiedade. Em contrapartida, menciona-se a jardinagem, atividade de manipulação de espécies vegetais em determinado espaços, sejam de caráter de ornamentação ou alimentícios, como as hortas comunitárias, com objetivo primordial de desenvolver lugares terapêuticos ${ }^{(8)}$. Além disso, grupos que praticam tal atividade têm maior tendência à mudança no estilo de vida e conscientização sobre o autocuidado físico e psíquico ${ }^{(9)}$. Em outras palavras, as alternativas de baixo custo e alta eficácia, como é o caso das ocupacionais, têm se tornado destaque no cenário científico e assistencial em saúde. Desta forma, objetivou-se relatar a experiência da implementação de uma horta comunitária, em uma Instituição de Longa Permanência para Idosos na capital cearense.

\section{MÉTODO}

Estudo na modalidade relato de experiência sobre a implementação de uma horta comunitária, em uma Instituição de Longa Permanência para Idosos, localizada na cidade de Fortaleza, Ceará, Brasil, em 2018. A atividade foi desenvolvida na disciplina Saúde do Idoso, pertencente ao Curso de Graduação em Enfermagem, da Universidade da Integração Internacional da Lusofonia Afro-Brasileira (UNILAB), sob a categoria de estágio supervisionado.

O cenário da ação foi o Lar de Amparo Aconchego ao Idoso Santa Terezinha, classificado como uma instituição não governamental, composta por 39 idosas do sexo feminino, nos mais variados grau de dependência. Historicamente, foi fundada por um sócio majoritário que por motivos pessoais de suprir uma perda familiar, fundou o lar em homenagem à mãe que leva o mesmo nome da referida ILPI.

O quadro de profissionais é formado por 24 integrantes, os quais incluem cuidadores, técnicos e auxiliares de enfermagem, cozinheiros, auxiliares de serviço gerais, médico, fisioterapeuta e assistente social, além do gerenciamento institucional. A rotina do Lar Santa Terezinha é basicamente integral, de modo que há rodízio de profissionais para $o$ atendimento a esses idosos que permanecem vinte e quatro horas na instituição.

Os familiares têm total liberdade de visita às idosas, ação incentivada pelo corpo administrativo, de modo a não perder o vínculo com os parentes, já que, de certa forma, o lar não é um local de abandono, mas um estabelecimento preparado para atender às necessidades dos moradores, com intuito residencial. Como toda instituição ILPI, também possui regras, como passeios que somente podem acontecer mediante acompanhamento por um familiar ou responsável, não é permitido parceiro do sexo masculino dentro da instituição, cada quarto pode comportar 
até três idosas, há horários definidos para cada refeição, as idosas não devem ficar retraídas no quarto, incentiva-se a socialização.

A princípio, com relação à ação propriamente dita, a ideia surgiu em visita anterior ao local que, por meio da observação, das conversas e percepções, notou-se situação de monotonia quanto aos momentos de lazer das idosas residentes. Em complemento, a estrutura contava com espaço inutilizável, próximo ao pátio social, que por iniciativa do gerenciamento em aderir à proposta, utilizou-se por meio de acordo prévio.

No que tange a execução da horta propriamente dita, o desenho e planejamento foi realizado por meio da cooperação entre o projeto formulado pelos acadêmicos de agronomia e a adaptabilidade dos tipos de alimentos a serem produzidos especificamente para o consumo da parcela geriátrica residente na instituição. $O$ desenho espacial da horta comunitária, assim como preparação do terreno, colheita das mudas, transferência, implantação, adubação e suplementação foram desempenhados pela Incubadora Tecnológica de Economia Solidária (INTER$\mathrm{SOL}$ ), classificado como um projeto de extensão continua vinculado a própria UNILAB.

Os alimentos escolhidos foram baseados e embasados conforme informações contidas no Manual de EnveIhecimento Ativo ${ }^{(10)}$, o qual preconiza um direcionamento quanto à dietas adaptativas para o público idosos com base em necessidades pertinentes da própria faixa etária. Na horta em questão foram cultivados mudas de cenoura, salsa, coentro, cebolinha e pepino. Todos os produtos mencionados se caracterizam por serem ricos em nutrientes tão necessário na ingestão cotidiana.

\section{RESULTADOS E DISCUSSÃO}

\section{Parceria multiprofissional e importância da im- plementação da horta comunitária}

A ação contou com a interação interdisciplinar entre - Curso de Enfermagem e Agronomia da mesma instituição educacional. Os participantes da equipe implementadora foram constituídos por dez acadêmicos, preceptora de estágio e gerencia da ILPI. A horta foi cultivada por algumas espécies vegetais, com fins alimentícios, sem uso de agrotóxicos ou pesticidas naturais que pudessem ocasionar algum dano à saúde dos manipuladores ou futuros consumidores.

As estratégias multiprofissionais na implementação de projetos tendem a funcionar de forma eficaz, em prol de um objetivo em comum, como neste estudo, fato que se estabelece pela variação de conhecimento e junção de áreas, a fim de desenvolver competências frente aos desafios $^{(11)}$. Por conseguinte, o paradoxo na formação acadêmica estimula o pensar diferente, com base em situações variadas, como foi a medida de unir a promoção da saúde e a realização de atividades ocupacionais na busca pelo bem-estar.

O primórdio da proposta surgiu por meio do diálogo com o setor gerencial da ILPI, como também pela observação integral durante o estágio, pautado principalmente na necessidade de espaço recreativo e, ao mesmo tempo, produtivo, a fim de beneficiar os moradores. Outro aspecto importante foi a comunicação efetiva com a equipe assistencial sobre contribuições que a universidade pudesse estar realizando, com vista a promover melhor qualidade de vida. Para isso, a iniciativa foi além do aspecto saúde, uma vez que trabalhar com organização estrutural, necessitaria basicamente da contribuição de pessoal específico da área.

Os dois estudantes de Agronomia convidados a executar a horta comunitária eram membros da INTERSOL, vinculada ao Instituto de Desenvolvimento Rural (IDR) da UNILAB. Esse grupo de extensão tem por missão promover o desenvolvimento de técnicas agrícolas voltadas para agricultura familiar e produtores locais. Notou-se, portanto, aproximação da obtenção do conhecimento científico adquirido em academia e aplicabilidade na realidade local, com ênfase na colaboração efetiva.

Em virtude dos fatos, a horticultura comunitária, em meio urbano, estimula movimentos coletivos quanto ao desenvolvimento econômico, ecológico e social, de modo que, prioritariamente, parte da visão sustentável em inserir a comunidade na participação efetiva da autoprodução alimentícia ${ }^{(12)}$. Nesse paradoxo, a junção de áreas distintas do conhecimento almeja resultados promissores e amplos, em que, na ação mencionada, nessa experiência, houve a descoberta de ferramenta modificadora de saúde, além do incentivo à postura ambiental responsável.

Outra vertente se estabelece quanto à implementação de iniciativa contínua em relação à aplicação ao treinamento da manutenção de hortas comunitárias. Os acadêmicos de Agronomia possibilitaram as devidas orientações aos funcionários da ILPI, em que se ressaltaram a manipulação adequada e o baixo custo na aquisição do sistema. $O$ investimento em tecnologias verdes ainda permanece bastante tímido, no que concerne às mudanças de conscientização ambiental, saúde nutricional e paisagismo racional( ${ }^{(13)}$.

O consumo de produtos orgânicos tem sido pauta de discussão na atualidade, inclusive na terceira idade, quanto ao envelhecimento saudável. Em alguns casos, as comorbidades delimitam a dieta, devido aos fatores de riscos e à pré-disposição a agravamentos. No Lar Santa Terezinha, parte considerável das idosas foram diagnosticados com hipertensão arterial sistêmica e diabete mellitus, com os devidos acompanhamento. Concomitante, a produção do próprio alimento corrobora a importância de refeições 
corretas, como também a educação dos participantes, por meio da prática da horticultura.

A alimentação regular reflete diretamente no funcionamento adequado do organismo, o que condiz com mudanças na qualidade de vida, nos quesitos físico e mental. Para isso, frutas, vegetais e alimentos ricos em proteínas auxiliam a baixa incidência de doenças cardíacas e perda e memória, problemas tão presentes entre a população da terceira idade ${ }^{(14)}$. A produtividade da horta, em meio ao convívio na ILPI, expõe a realidade ao incentivo à sustentabilidade e aquisição de produtos de origem confiável para ingestão no dia a dia.

Portanto, as diferentes visões em torno de um mesmo problema expandiram as opiniões e a busca constante para resolutividade, em consonância com a equipe multiprofissional. Desta forma, o canteiro serviu não somente para unir a ciência da rotina cotidiana, como também para expandir novos horizontes, em meio à abordagem interdisciplinar, além de consolidar tecnologias otimizadores, de acordo com o perfil e particularidade de cada situação e contexto.

\section{Importância de atividades lúdicas em ILPI}

Diversos são as razões para que idosos se instalem em uma ILPI, desde questões familiares até fatores socioeconômicos. Diante do pressuposto, essas instituições constituem verdadeiros lares coletivos, em prol do acolhimento, com base na necessidade de cada grupo. A experiência vivenciada permitiu conhecer a personalidade dos moradores, histórias e sonhos compartilhados com os profissionais e demais colegas residentes.

Contrariamente a alguns preceitos construídos pela sociedade, como abandono e solidão, os participantes do Lar Santa Terezinha se mostraram contentes com o tipo de assistência prestada, de modo a prevalecer os direitos, em âmbito social e coletivo, com respaldo no poder decisório. O livre arbítrio da pessoa idosa possui representações significativas, com a finalidade de melhorar a qualidade do cuidado e adquirir atuação participativa na prática de gestão(15).

De forma mais ampla, as instalações da ILPI lócus deste estudo se enquadravam em um ambiente adequado para abrigar as idosas, porém, não existia cenário lúdico propriamente dito, o que se remetia a apenas um pátio que esporadicamente era utilizado por estudantes e visitantes na promoção de algum evento. As atividades ocupacionais eram restritas a rodas de conversas, ao final da tarde, ou ações realizadas pela assistente social, integrante da equipe de assistência.

Detectou-se, também, a participação efetiva em tarefas realizadas pelos acadêmicos de Enfermagem, o que motiva a reflexão acerca da importância do incentivo em inserir o idoso em dinâmicas interativas. A horta co- munitária se tornou alterativa eficaz, por transformar o ambiente e integrar o participante no processo de manutenção, além de indicar relações positivas, baseadas na horticultura doméstica e no estilo de vida saudável(16). Aproximar pessoas da jardinagem desperta concepções e razões para o viver sob inúmeros aspectos.

Outro benefício foi o paisagismo verde, inserido em meio aos muros de concreto da instituição, cuja finalidade foi promover espaço mais arborizado, de modo a proporcionar ambiente de convivência mais agradável e, ao mesmo tempo, produtivo, com fins alimentícios. Os recintos de lazeres não são muito comuns nas ILPI, seja por limitação ou deficiência física motora, ou pelo lado emocional e cognitivo ${ }^{(17)}$. Nesse particular, as adaptações incrementam a acessibilidade, uma vez que ajusta os limites do indivíduo e o integra, em virtude da equidade.

O lúdico exerce papel fundamental no rompimento da monotonia, promove a interação e o despertar para postura ativa na promoção do autocuidado. Diante dos fatos, a pessoa com demência, patologia presente na terceira idade, possui mais limitação, no que tange à interação social. No entanto, as práticas lúdicas despertam condições necessárias no imaginário do indivíduo envolvido que, por meio da imaginação e criatividade, alcançam a confiança na realização de ocupações e equilíbrio mental(18).

Nesse sentido, a horta implementada vislumbrou mudanças na dinamicidade do Lar Santa Terezinha, embora tenha ficado como contribuição final do estágio realizado. Em síntese, a inciativa proporciona discussão acerca da necessidade da inserção constante de atividades lúdicas com idosos institucionalizados, em que há ganho quanto à qualidade de vida e ao entrosamento entre estudantes, equipe e moradores dessas entidades.

Já as dificuldades mínimas podem ser consideradas mínimas, de maneira a serem vinculadas a decisões burocráticas quanto a autorização de implementação e a falta de jardineiros, o que foi reduzido consequentemente por meio de treinamentos e orientações realizados pela INTERSOL com os próprios funcionários efetivos da instituição. Ao corroborar com esse tipo de decisão, o surgimento da proposta até a realização exige um estabelecimento de confiança entre os promotores e a esfera gerencial desse tipo de instituição, uma vez que a inserção desse tipo de projeto altera de forma positiva a rotina da ILPI.

\section{CONCLUSÃO}

De maneira geral, a implementação da horta comunitária na Instituição de Longa Permanência para Idosos contribuiu de forma significativa no contexto lúdico e fins alimentícios, tendo em vista o aproveitamento de espaço inutilizável, em consonância com a rotina monótona dos 
moradores. Portanto, esse tipo de iniciativa se torna aplicável, devido ao baixo custo de instalação e investimento quanto à produção de produtos orgânicos, mediante a horticultura doméstica.

Além disso, perceberam-se benefícios em relação à parceira multiprofissional e interdisciplinar na resolutividade de questão sociais, como o caso da promoção da saúde. Nesse quesito, o aprendizado durante o estágio, juntamente à realização de projetos viáveis, em campo prático, contribuiu para formação acadêmica mais diferenciada e, ao mesmo tempo, próxima da realidade, com base na experiência vivenciada. Coloca-se futuras investigações principalmente na adaptabilidade das ILPI brasileiras para implementar esse tipo de projeto 


\section{REFERÊNCIAS}

I. Bassler TC, Santos FR, Santos Junior AC, Furlan MCR, Maia $\mathrm{CR}$. Avaliação da qualidade de vida de idosos residentes em instituição de longa permanência para idosos. Rev. enferm. UFPE on line [Internet] 2017. [Acesso $2020 \mathrm{dez}$ 30]; II(I):10-17. Available from: https://pesquisa.bvsalud. org/portal/resource/pt/bde-30266

2. Camarano AA, Kanso S.As instituições de longa permanência para idosos no Brasil. Rev Bras Estud Popul [Internet]. 2010. [Acesso 2020 ago 28];27(I):232-5. Available from: http:// dx.doi.org/10.1590/S0102-309820100001000I4

3. Alves MB, Menezes MR, Felzemburg RDM, Silva VA, Amaral $\mathrm{JB}$. Instituições de longa permanência para idosos: aspectos físico-estruturais e organizacionais. Escola Anna Nery [Internet] 2017. [Acesso 2020 ago 28];2I(4). Available from: https://doi.org/ I0.1590/2 I77-9465-ean-2016-0337

4. Brasil. Ministério da Saúde. Resolução da Diretoria Colegiada - RDCI ANVISA N²83, de 26 de setembro de 2005. Brasília (DF): Ministério da Saúde [Internet] 2005. [Acesso 202025 ago 28]. Available from: http://portal.anvisa.gov.br/ documents/I018I/27/8376/RDC_283_2005_COMP.pdf/ a38f2055-c23a-4eca-94ed-76fa43acb I df

5. Artmann M, Chen X, lojă C, Hof A, Onose D, Poniży L, Lamovšek AZ, Breuste J. The role of urban green spaces in care facilities for elderly people across European cities. Urban Forestry \& Urban Greening [Internet] 2017. [Acesso 2020 ago 15];27:203-2I3. Available from: https:// doi.org/10.1016/j.ufug.2017.08.007

6. Mugo JW, Onywera V,Waudo J, Otieno OG. Functionality of Elderly Persons: A Comparison Between Institutionalized and Non - Institutionalized Elderly Persons in Nairobi City County, in Kenya. World Journal of Public Health [Internet] 20I8.[Acesso2020ago I3];3(I):9-I5Availablefrom:file:///C:I Users/Dell/Downloads/I0.I I648.j.wjph.2018030I.I2.pdf

7. Jerez-Roig J, Oliveira NPD, Filho BFL, Farias MA, Matias BMGL, Ferreira LM, Amaral FLJS, Souza DLB, Lima EC. Depressive Symptoms and Associated Factors in Institutionalized Elderly. Taylor e Francis Online [Internet] 2016. [Acesso 2020 ago I5]. Available from: https://doi. org/l0.1080/0361073X.2016.1224673

8. Pitt H. Therapeutic experiences of community gardens: putting flow in its place. Health \& Place [Internet] 2014. [Acesso 2020 ago 14];27:84-9I. Available from: https://doi. org/10.1016/j.healthplace.2014.02.006

9. Joyce J, Warren A. A Case Study Exploring the Influence of a Gardening Therapy Group on Well-Being. Taylor e Francis
Online[Internet]20 I6.[Acesso2020ago 19];203-2I5Available from: https://doi.org/I0.1080/0164212X.2015.1 I I I 84

10. Ribeiro O, Paúl C. Manual de envelhecimento activo. 2. ${ }^{a}$ Edição. Editora. Lisboa: Lidel. 201 I.

II. Silva RS, Fedosse E, Pascotinia FS, Riehsc EB. Condições de saúde de idosos institucionalizados: contribuições para ação interdisciplinar e promotora de saúde. Cad. Bras. Ter. Ocup [Internet] 2019. [Acesso 2020 ago I8];27(2):345-356.Available from: https://doi.org/10.4322/2526-8910.ctoAOI590

12. Rogge N, Theesfeld I, Strassner C. Social Sustainability through Social Interaction-A National Survey on Community Gardens in Germany. Sustainability [Internet] 2018. [Acesso 2020 ago 25]; 10:1085. Available from: https://doi.org/10.3390/sul004I085

I3. Egli V, Oliver M, Tautolo E. The development of a model of community garden benefits to wellbeing. Preventive Medicine Reports [Internet] 2016. [Acesso 2020 ago 25];(3):348-352. Available from: https://doi.org/10.1016/j. pmedr.2016.04.005

14. Xu X, Ling M, Inglis SC. et al. Eating and healthy ageing: a longitudinal study on the association between food consumption, memory loss and its comorbidities. Int J Public Health [Internet] 2020. [Acesso 2020 ago 25];65:57I-582. Available from: https://doi.org/10.1007/s00038-020-01337-y

I5. Tuominen L, Leino-Kilpi H, Suhonen R. Older people's experiences of their free will in nursing homes. Nursing Ethics [Internet] 20 I6. [Acesso 2020 ago I 2];(23): I .Available from: https://doi.org/I0.I I77/0969733014557II9

16. Machida D. Relationship between Community or Home Gardening and Health of the Elderly: A Web-Based CrossSectional Survey in Japan. Int. J. Environ. Res. Public Health [Internet] 2019. [Acesso 2020 ago 26]; I6(8): I 389.Available from: https://doi.org//0.3390/ijerph I6081389

17. Raymond É, Tremblay C, Ruest R, Lebel JG. Access to leisure environments for seniors with disabilities: $A$ guide to developing a practical guide.: Loisir et Société [Internet] 2019. [Acesso 2020 ago 16];42(I): I2I-136. Available from: https:// www.cabdirect.org/cabdirect/abstract/2019327I88I

I8.Tsekleves E, Bingley AF, Escalante MAL, Gradinar A. Engaging people with dementia in designing playful and creative practices: Co-design or co-creation? Dementia [Internet] 2020. [Acesso 2020 ago 25];19(3). Available from: https:// doi.org/10.1177/1471301218791692

Recebido: 2020-10-15

Aceito: $2020-1 \mid-27$ 